\title{
Stoma-Closure-Induced
}

\section{Fulminant Pseudomembranous} Colitis Recovered by Adjunctive Intracolic Vancomycin with Postural Change

\author{
Yozo Suzukia,c Keiichi Takahashi ${ }^{a}$ Tatsuro Yamaguchi ${ }^{a}$ \\ Hiroshi Matsumoto $^{a}$ Daisuke Nakano ${ }^{a}$ Koichi Koizumi ${ }^{b}$ \\ Departments of a Surgery and ${ }^{b}$ Gastroenterology, Tokyo Metropolitan Cancer and \\ Infectious Diseases Center, Komagome Hospital, Tokyo, and 'Department of \\ Gastroenterological Surgery, Osaka University Graduate School of Medicine, \\ Osaka, Japan
}

\section{Key Words}

Clostridium difficile - Pseudomembranous colitis - Intracolic vancomycin therapy . Postural change

\begin{abstract}
A 67-year-old man with a history of low anterior resection and diverting loop transverse colostomy for rectal carcinoma developed fulminant pseudomembranous colitis after stoma closure. Oral administration of vancomycin at $0.5 \mathrm{~g}$ every $6 \mathrm{~h}$ and colonoscopy with intracolic vancomycin administration was unsuccessful, but continuation of intracolic vancomycin with postural change resulted in dramatic recovery. Postural change may extend the efficacy of intracolic vancomycin, and intracolic vancomycin should be considered as an option between conventional therapy and surgical intervention for pseudomembranous colitis.
\end{abstract}

\section{Case Report}

The patient, a 67-year-old male with a previous medical history of hypertension and ossification of posterior longitudinal ligament of cervical spine, underwent low anterior resection with diverting loop transverse colostomy for rectal carcinoma at Tokyo Metropolitan Cancer and Infectious Diseases Center, Komagome Hospital in 2006. Since the anal margin was positive, postoperative radiotherapy was added ( 50 Gy per 25 fractions). Endoscopic dilation of anastomosis was performed, followed by stoma closure with resection and hand-sewn anastomosis 10 months after low anterior resection. At laparotomy, cefmetazole $2 \mathrm{~g}$ was administered. The patient's recovery was uneventful until postoperative day 8 when he developed high fever $\left(38.8^{\circ} \mathrm{C}\right)$, mild hypoxia and distension without 
diarrhea. White blood cell (WBC) count was 18,300 cells $/ \mathrm{mm}^{3}$ and C-reactive protein (CRP) was $12.8 \mathrm{mg} / \mathrm{dl}$. Administration of oxygen and parenteral cephoperazon/sulbactam (CPZ/SBT) $0.5 \mathrm{~g}$ twice daily were started empirically. On postoperative day 9 , computed tomography of the abdomen showed the dilated (up to $59 \mathrm{~mm}$ ) colon with thickened (up to $3 \mathrm{~mm}$ ) wall. Colonoscopy showed no stricture of anastomoses, but revealed aperistalsis and diffuse pseudomembranes (fig. 1a), which resulted positive for Clostridium difficile toxin A by immunochromatography assay (UNIQUICK, Kanto Chemical Co., Inc., Tokyo, Japan).

The CPZ/SBT was discontinued and a course of oral vancomycin $500 \mathrm{mg}$ and live probiotic Bifidobacterium $1 \mathrm{~g}$ respectively four times daily were started for fulminant pseudomembranous colitis (PMC). Though flatus and defecation were seen, distention exacerbated on the fifth day of therapy. Colonoscopy revealed exacerbation of PMC (fig. 1b), and a soft 22-French catheter was placed in the colon. The tip was positioned in the transverse colon. During the colonoscopy, decompression and vancomycin $500 \mathrm{mg}$ was administered directly into the colon. It was followed with intracolic administration of vancomycin $500 \mathrm{mg}$ with live probiotic Bifidobacterium $1 \mathrm{~g}$ in $500 \mathrm{ml}$ water every six hours. The retention time of enema was one hour. A 12-French transnasal ileus tube was also placed. On the 20th day of the therapy (15 days after the initiation of intracolic vancomycin), he had high fever $\left(39.5^{\circ} \mathrm{C}\right.$ ), though exacerbation of inflammation was not evident (WBC 5,700 cells $/ \mathrm{mm}^{3}, \mathrm{CRP} 9.6 \mathrm{mg} / \mathrm{dl}$ ), and a third colonoscopy was performed. It showed partial improvement and partial exacerbation of PMC. In the improved portion, edematous regenerative mucosa was seen, and the location included ventral cecum and transverse colon (fig. $2 \mathrm{a}$ ). In the exacerbated portion, some ulcers with exposed muscularis propria were seen with coagula, and the location was limited to the dorsal cecum and hepatic flexure (fig. 2b). It seemed to implicate that the dorsal mucosa was severely damaged and that there was ventral improvement, so we changed the pattern of drug delivery, changing the patient's position every $15 \mathrm{~min}$ (spine, right decubitus, prone, left decubitus) in order that the intestinal fluid and the medicine were mixed without staying at the same place, which resulted in gradual clinical improvement. A fourth colonoscopy was performed after 13 days of the new pattern of intracolic vancomycin administration, and it showed complete resolution of the colitis without detectable pseudomembranes, though some ulcer scars were seen (fig. 3 ). The ileus tubes were removed 30 days after the initiation of vancomycin. The patient suffered stricture of esophagogastric junction, which was resolved by endoscopic dilation, and has been symptom-free so far.

\section{Discussion}

Closure of temporary stoma is known to have a set of potential surgical complication, such as leak, wound infection, stricture, obstruction and hernias [1], but C. difficile fulminant colitis after closure of temporary stoma has never been reported. Radiotherapy has been reported to have a chance to increase $C$. difficile, in the setting of patients with uterine carcinoma who underwent postoperative radiation therapy [2], but this patient was free of symptom during and after radiotherapy.

The clinical picture of a C. difficile infection varies from an asymptomatic carrier to fulminant colitis with toxic megacolon. Age over 65 year, previous antibiotic use, immunosuppression, acid suppression, and operation are reported to increase the incidence of $C$. difficile-associated disease (CDAD) [3, 4], and a WBC count $>16,000$ cells $/ \mathrm{mm}^{3}$ at initiation of therapy, operative therapy within the last 30 days, a history of inflammatory bowel disease, and a history of intravenous immunoglobulin treatment are risk factors for the development of fulminant C. difficile colitis [5].

Treatment of CDAD starts with discontinuation of offending antibiotics and supportive therapy. For those who faile to respond within 2-3 days, the addition of metronidazole is recommended, and vancomycin given orally is reserved for patients who fail to respond to metronidazole, whose organism is resistant to metronidazole, who are unable to tolerate metronidazole, who are pregnant, who are children under the age of 10 , or who are critically ill [6]. Since our patient was critically ill, we adopted vancomycin. 
Surgical intervention is considered an option for CDAD patients with toxic dilatation of the colon, bowel perforation, systemic toxicity, and failure to respond to medical treatment, but postoperative mortality is as high as $40 \%$ [3]. Since the patient was critically ill but vitally stable, we decided to continue medical treatment. Intravenous metronidazole can be an option for critically ill patients who are unable to take oral antimicrobial agents [7], but its efficacy is controversial [8] and it is not available in Japan. So we selected another option, intracolic vancomycin therapy [9]. The reported regimen varies (dose 1-3 g/day, interval 4-12 h), and the concerns about the potential efficacy of delivery of intracolic vancomycin to the transverse and right colon has been discussed $[9,10]$, and it has never been directly shown by colonoscopic imaging before.

This case is unique in that the delivery of intracolic vancomycin was achieved by transanal ileus tube with postural change, and in that the time course of exacerbation from left-side colitis to diffuse colitis and its recovery was recorded with frequent colonoscopic studies. It is notable that vancomycin reached the cecum through the transanal ileus tube, which was indicated by the improvement of dorsal cecum shown by the $3 \mathrm{rd}$ colonoscopy. It is also notable that the findings indicate that the right colon, especially the dorsal end, can be the critical portion for treatment failure. This may be related to the obligate anaerobic nature of $C$. difficile [11] and the postural change may help increase the exposure of oxygen to $C$. difficile that hide beneath the intestinal fluid.

Though the time course was longer than in other reported cases (2-14 days) [9], this patient was barely controlled. The frequent decompression and survey by colonoscopy, followed by continuous decompression and intracolic administration of vancomycin through the transanal long tube with postural change, should be considered an option between conventional therapy and surgical intervention for PMC.

Fig. 1. Colonoscopy performed on postoperative day 9 revealed diffuse areas of pseudomembranes throughout the remaining colon (a), and colonoscopy performed after 5 days of oral vancomycin revealed exacerbation of colitis (b).

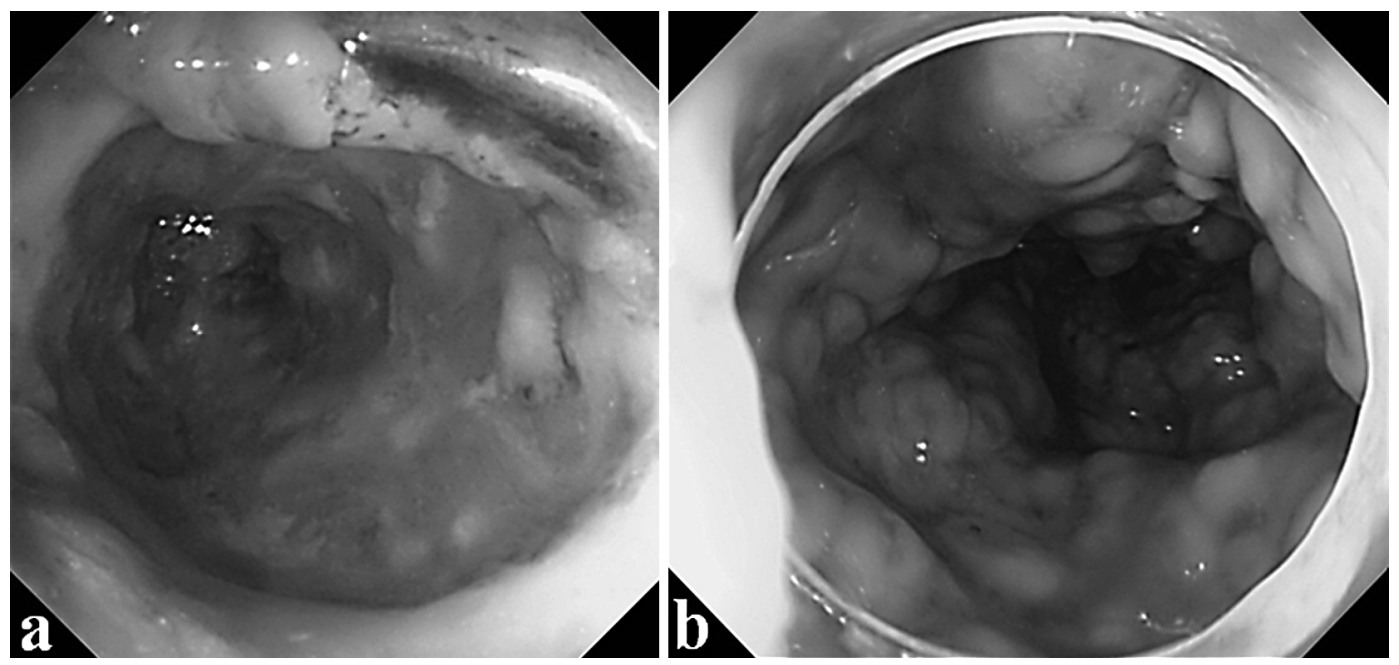


Fig. 2. Colonoscopy performed 15 days after the second colonoscopy, during which intracolic and transnasal vancomycin was administered, revealed the mixture of exacerbation and improvement. The cecum consisted of an exacerbated dorsal portion and an improved ventral portion (a), and exacerbation was severe especially around the hepatic flexure (b). In the improved portion, mucosa was very edematous, and in the exacerbated portion, some ulcers with exposed muscularis propria were seen with coagula.
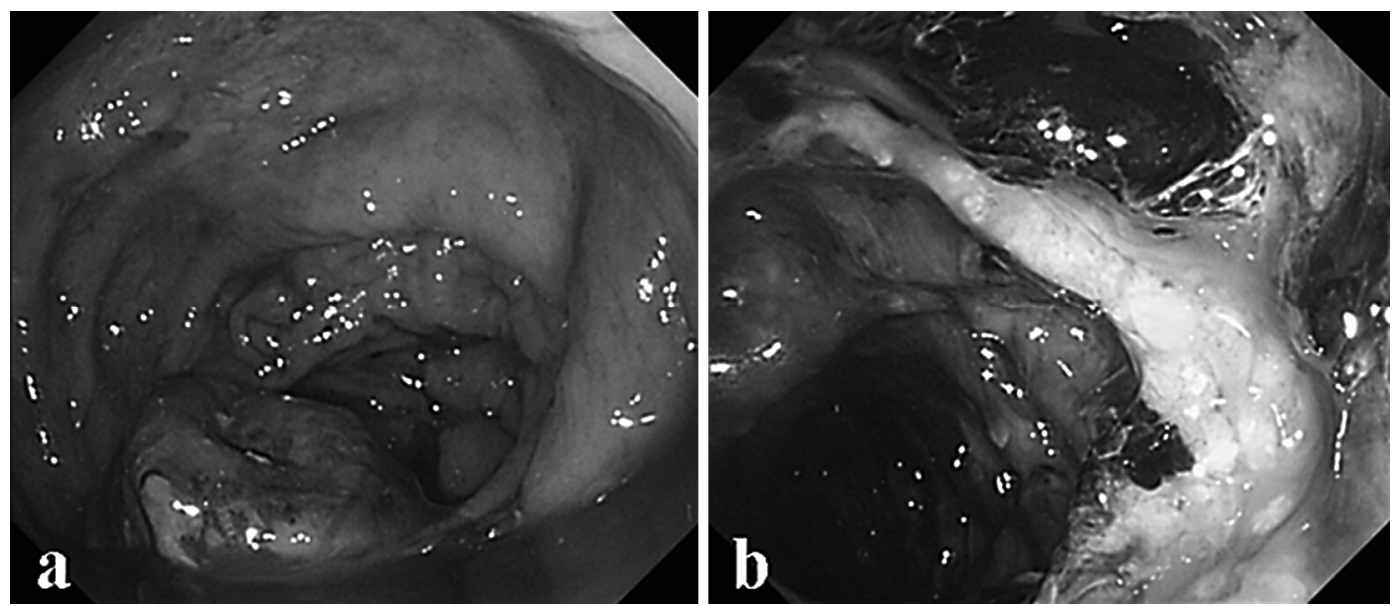

Fig. 3. Colonoscopy performed 13 days after the third colonoscopy, during which postural change during intracolic vancomycin administration was performed, showed complete resolution of the colitis without detectable pseudomembranes (a), though some ulcer scars were seen in the dorsal cecum and the hepatic flexure (b).
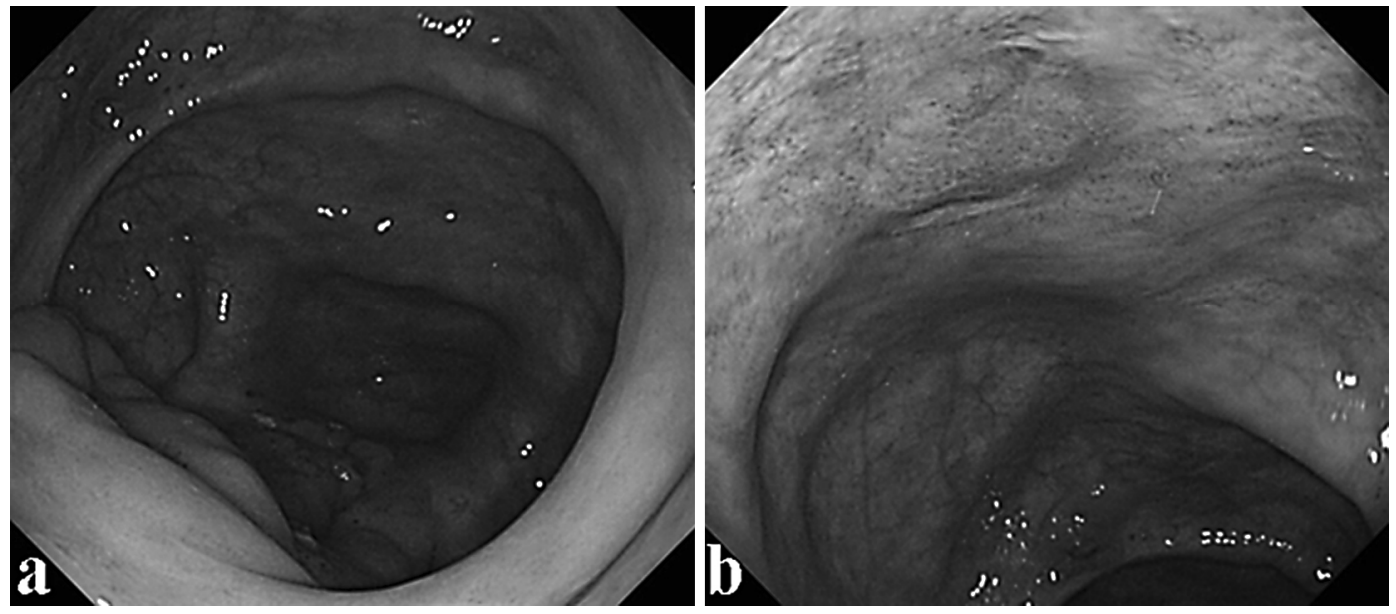


\section{References}

1 Shellito PC: Complications of abdominal stoma surgery. Dis Colon Rectum 1998;41:1562-1572.

2 Cuzzolin L, Zambreri D, Donini M, Griso C, Benoni G: Influence of radiotherapy on intestinal microflora in cancer patients. J Chemother 1992;4:176-179.

3 Monaghan T, Boswell T, Mahida YR: Recent advances in Clostridium difficileassociated disease. Gut 2008;57:850-860.

4 Cleary RK: Clostridium difficile-associated diarrhea and colitis: clinical manifestations, diagnosis, and treatment. Dis Colon Rectum 1998;41:1435-1449.

5 Greenstein AJ, Byrn JC, Zhang LP, Swedish KA, Jahn AE, Divino CM: Risk factors for the development of fulminant Clostridium difficile colitis. Surgery 2008;143:623-629.

6 Fekety R: Guidelines for the diagnosis and management of Clostridium difficileassociated diarrhea and colitis. American College of Gastroenterology, Practice Parameters Committee. Am J Gastroenterol 1997;92:739-750.

7 Friedenberg F, Fernandez A, Kaul V, Niami P, Levine GM: Intravenous metronidazole for the treatment of Clostridium difficile colitis. Dis Colon Rectum 2001;44:1176-1180.

8 Guzman R, Kirkpatrik J, Forward K, Lim F: Failure of parenteral metronidazole in the treatment of pseudomembranous colitis [letter]. J Infect Dis 1988;158:11461147.

9 Apisarnthanarak A, Razavi B, Mundy LM: Adjunctive intracolic vancomycin for severe Clostridium difficile colitis: case series and review of the literature. Clin Infect Dis 2002;35:690-696.

10 Pasic M, Jost R, Carrel T, Von Segesser L, Turina M: Intracolic vancomycin for pseudomembranous colitis. N Engl J Med 1993;329:583.

11 Hall IC, O'Toole E: Intestinal flora in new-born infants with a description of a new pathogenic anaerobe: Bacillus difficilis. Am J Dis Child 1935;48:390-402. 